Journal of Engineering and Applied Sciences 10 (5): 110-117, 2015

ISSN: 1816-949X

(C) Medwell Journals, 2015

\title{
Technique of Computer-Aided Design of Power Supply System of Vehicles
}

\author{
Evgenii Yu. Fedorov, Andrey V. Ferenetz and Valery S. Tereshchuk \\ Department of Electrical Equipment, \\ Kazan National Research Technical University named after A.N. Tupolev-KAI, Kazan, Russia
}

\begin{abstract}
The study describes a technique of computer-aided design of Vehicle Power Supply System. Algorithms of layout and placement of electrical equipment elements, algorithm for bunched wiring in computer-aided design have been developed.
\end{abstract}

Key words: Vehicle, computer-aided design, power supply system, optimization, bunched wiring

\section{INTRODUCTION}

Design of power supply system of modern vehicles now is not possible without use of computer-aided systems for the following reasons: a number of new models, modifications and complete sets of vehicles constantly grows; strict requirements are charged to timing and quality of project documents their compliance with international standards and regulations; the structure of power supply system is being complicated which leads to increase in the range and complexity of the drawings.

In the world engineering, a tendency of ensuring the close relationship of design, production, marketing and maintenance of the vehicle is observed. Therefore, the design of power supply system of the vehicle is inseparable from further technological preparation of production and maintenance. The use of Computer-Aided Design (CAD) based on CALS-technologies is relevant, consisting in continuous information support of supply and life cycle of complex products (design, production, maintenance, repair, recycling).

The market of information technologies in the field of design of power supply system is widely represented by multi-purpose tool CAD systems, allowing us to solve the complex task of designing electrical schematic circuits, wiring diagrams and designing the bunch wiring. As example such CAD systems may be referred as Electrics, E3. Series, EPLAN Electric and others. Most of them effectively interact with three-dimensional design systems (Siemens NX, Catia, Simatron, Solid Works), systems of preparation and maintenance of technical documentation (TG BILDER) and systems for organization of collective use (Teamcenter, Smartteam, CIM Database).

For problems solved by development departments of electric equipment of vehicles the level of project is determined by the level of reliability and quality of electricity supply to consumers of electricity of existing systems and optimality of decisions made. These include the layout and arrangement of structural units, electrical circuits wiring, forming the bunches, analysis of operation modes of power supply system, optimization of wire cross sections, selection and verification of protection devices, electrical energy balance calculation of the vehicle, etc.

The performed analysis of computer-aided design and optimization of systems of vehicles power supply has shown that the systems of computer-aided design of electrical equipment are mostly graphical and information tools to support the design process significantly reduce the time and increase its accuracy. But the quality of the design decisions here depends largely on readiness and expertise of engineering staff (Norenkov, 2002).

The problems of optimization of analysis of quality and reliability of designed system are not solved by multi-purpose CAD tools of power supply system. In addition, the multi-purpose $\mathrm{CAD}$ being instrumental jacket, need to be adapted to specific objects, specifics of problem solving and form of design documentation.

Thus, to solve these problems, one should study the design stage, creation of algorithms to optimize the power supply system, development of specialized software, solving the problems of optimization as well as development of appropriate new and effective technique of computer-aided design of power supply system of vehicles.

\section{MATERIALS AND METHODS}

Main part: The process of designing a Vehicle Power Supply System consists of solving many problems. Figure 1 shows a model of the design stage of the vehicle power supply system in the form of block diagram. The basis of the design stage is development of schematic

Corresponding Author: Evgenii Yu. Fedorov, Department of Electrical Equipment,

Kazan National Research Technical University named after A.N. Tupolev-KAI, Kazan, Russia 


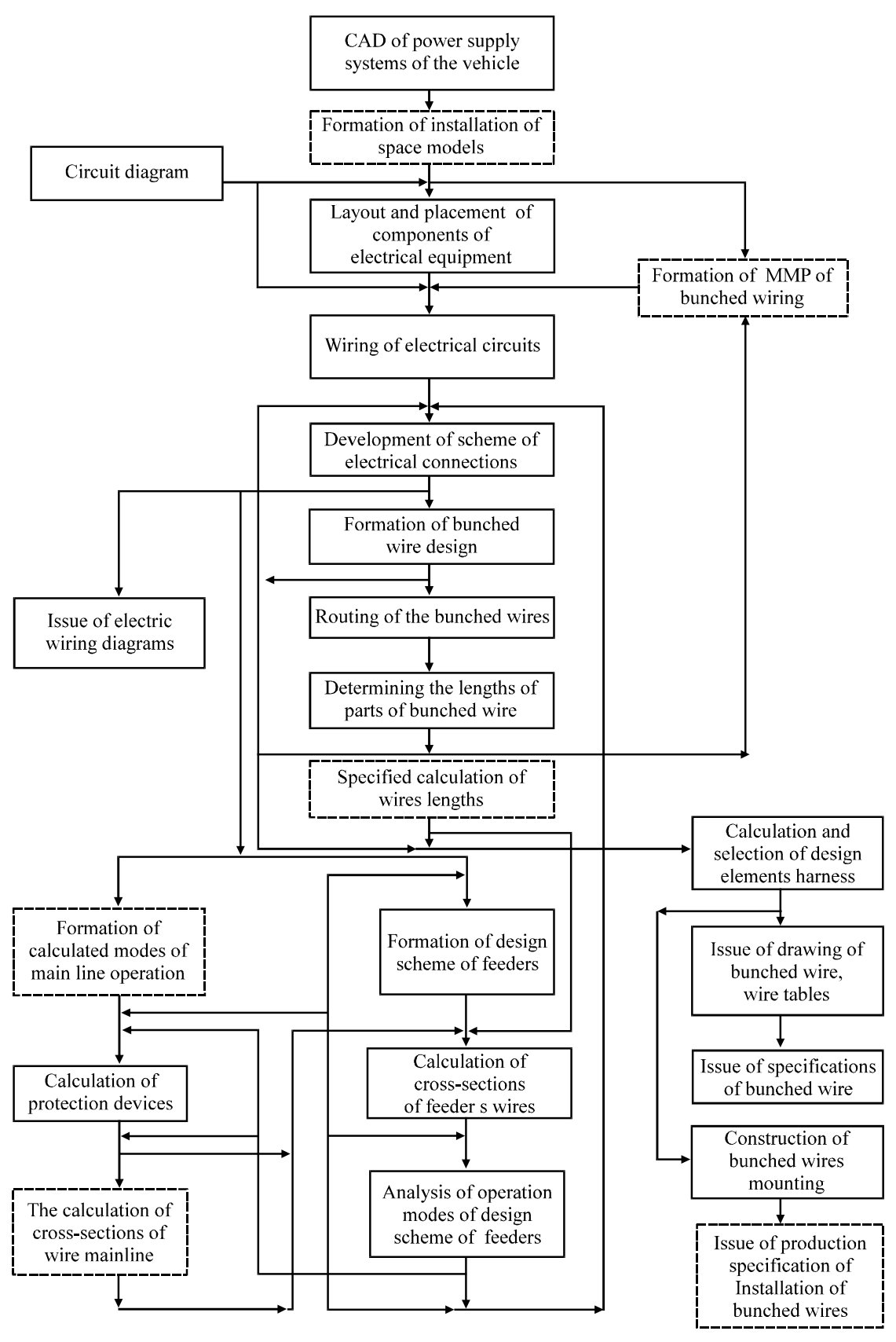

Fig. 1: Block diagram of the process of designing the power supply system

diagrams. The main labor input is the task of determining the spatial position of the elements their connections and preparation of corresponding technical documentation: tables of connections, specifications, etc. Such problems represent a class of topological design problems.

When designing the interblock assembling they are corresponded to the sequence of performing the tasks of layout and placement of structural assemblies, wiring of electrical circuits, forming and tracing the bunched cables.
In solving the problems of placing, determining the configuration of electrical network of the vehicle one must take into account the voltage losses in the wires and multi-mode operations in the network. This leads to the fact that the design of all electrical systems being interconnected that is the design process is of complex iterative character. Thus, the synthesis of the power supply system and its parameter optimization is the final stage in the design. 
Forming the sets (layout) of design units and their placing on the vehicle is the important stage. The placement of structural units, restrictions on their volume, etc. should be determined The results of decisions determine the future course of wiring the electrical circuits, forming and tracing the bunched cables. The problem of layout and location of structural assemblies is most commonly found in adjacent areas: designing the electronic equipment, automation devices. In these, works are widely used serial and iterative algorithms using the theory of undirected graphs and various methods of mathematical programming. As the criteria of optimization are used different estimates of the length of connections.

The wiring of electrical circuits is to determine a way of connecting the equipotential terminals of electric circuit and is conducted taking into account the routes and location of elements of each chain, the requirements of reliability, etc. Of known to researchers works (Fedorov et al., 2015) in them are represented numerous solutions of the problem of electrical circuits wiring in both aircraft, automobiles and in electronic equipment, computing equipment. Electrical circuits are represented by the graph models and the problem is reduced to determination of the minimum covering trees.

The task of forming the bunched wires is to integrate the sets of wires taking into account the limitations as to electromagnetic compatibility, current type, manufacturability, geometric features, places of wiring, etc. The number of works on bunched wires forming is limited, due to the difficulty of setting objectives for a significant number of design decisions. In the current works, the problem of forming the bunched wires is solved by applying the orthogonal graphs and algorithms for constructing the minimal trees.

The task of tracking the bunched wires is to build the optimal trajectory of bunched wires in the installation space of the automobile. The works are known which explore the tracing of complex connections and bunched wires for aircraft. In all the considered papers tracing of bunched wires is made in two dimensional space using the graph models (Hart et al., 1968; Lee, 1961). The tasks of parametric synthesis involve selecting the protection devices, optimization of wire cross sections, analysis of operational modes of the system of electric energy distribution, electric energy balance calculation of the vehicle.

Analysis of the structural scheme of design process of power supply system indicates that some operations (basic scheme, wiring diagram, etc.) can be implemented with the help of existing $\mathrm{CAD}$ systems. But the others (layout of electrical equipment, electrical circuits wiring, forming and tracing the bunched wires, calculations of currents, voltages, wire cross sections) are designed and adapted to the design process by the Department of Electrical equipment of KNRTU-KAI. Currently, the software of computer aided design of power supply system has been developed: the algorithms of layout and placement of structural units of electrical equipment, the algorithm of electrical circuits wiring, the algorithm of forming and tracing the bunched wires, wire cross sections optimization algorithm, the algorithm for choosing the protection devices, the algorithm of analysis of power supply system operation modes.

\section{RESULTS AND DISCUSSION}

Layout of electrical equipment: The task of electrical equipment layout is to bring together the elements in structural components. Today, strict requirements are charged to the weight and dimensions of vehicles related to the terms of fuel consumption, maneuverability, maintainability, aesthetic qualities, etc. Elements of electrical equipment (electromagnetic relays, contactors, circuit breakers and other switchgear) of vehicles are placed not orderly and chaotic, often according to residual free space.

Grouping the given elements in the structural components will allow one to provide additional volumes to reduce the dimensions and placement of additional equipment, etc.

The problem of layout is generally stated as follows: it is required to divide the set of assembly elements $\mathrm{E}$ by $\alpha$ disjoint subsets Es, being an element set of structural nodes.

When solving this problem many limitations $\mathrm{C}$ is formed by using elements of the variety of features of system model and the following engineering requirements:

- The requirements of electromagnetic, thermal, mechanical compatibility, reliability must be satisfied

- The predetermined mark-to-space ratio of structural units should be provided

- The amount of structural assemblies should be limited

The schematic circuit consisting of plurality of elements $\mathrm{E}=\left\{\mathrm{e}_{1}, \mathrm{e}_{2}, \ldots, \mathrm{e}_{\mathrm{n}}\right\}$ interconnected by plurality of electrical circuits $\mathrm{V}=\left\{\mathrm{v}_{1}, \mathrm{v}_{1}, \ldots, \mathrm{v}_{\mathrm{m}}\right\}$ will be called switching circuit. 
Each ith element has a plurality of connected terminals $\mathrm{C}_{1}=\left\{\mathrm{c}_{11}, \mathrm{c}_{\mathrm{i} 2}, \ldots, \mathrm{c}_{\mathrm{ik}}\right\}$. External outputs of the circuit, serving for connection with other schemes (for example, through the electric connector), it is convenient to represent by fictitious element $\mathrm{e}_{0}$.

The circuit diagram may be represented in the form of Graph of Switching Circuit (GSC). It contains three types of peaks corresponding to: E-components, C-terminals of the elements, $\mathrm{V}$-circuits (complexes). The ribs are divided into element $\mathrm{F}$ and signal ones $\mathrm{W}$ :

$$
\mathrm{G}=(\mathrm{E}, \mathrm{V}, \mathrm{C}, \mathrm{F}, \mathrm{W})
$$

The structure of GSC can be specified by one matrix $\mathrm{T}=\left\|\mathrm{t}_{\mathrm{i}}\right\|_{\text {se }}$, the rows of which correspond to the elements and columns to the output of element with:

$$
\mathrm{K}_{1}=\operatorname{maxk}_{\mathrm{i}}, \mathrm{i}=1, \mathrm{n}
$$

where, $\mathrm{n}$ number of elements. Matrix $\mathrm{T}$ is called circuit matrix. To construct the circuit matrix, the number must be assigned to each chain.

The most appropriate criterion is the criterion of minimizing the number of connections between the structural units as it gives weight reduction of products, minimizes mutual interferences, increases reliability, simplifies design. The disadvantage of this creation is that it does not take into account the weight and size of electrical connections, namely, the cross-section of the wires connecting the structural components.

For example as the result of layout problem solving two solutions were obtained (Fig. 2). It is seen from Fig. 2 that option 1 is preferred as to criterion of taking into account external relations but considering cross-sections of wires (let $S_{q 1}=4 \mathbf{M M}^{2}, S_{q 2}=1 \mathbf{M M}^{2}$ ), then the value of criterion according the option 1 :

$$
\mathrm{S}_{1}=3 \times \mathrm{S}_{\mathrm{q} 1}=12 \mathbf{M M}^{2}
$$

The value of the criterion as to option 2 :

$$
\mathrm{S}_{2}=4 \times \mathrm{S}_{\mathrm{q} 2}=4 \mathrm{MM}^{2}
$$

Thus, the preferred option is 2. In developed algorithm of layout of design nodes it is necessary to consider this factor by introducing the additional coefficient of circuit $k_{\mathrm{q}}$ which takes into account the cross-section of the wire.

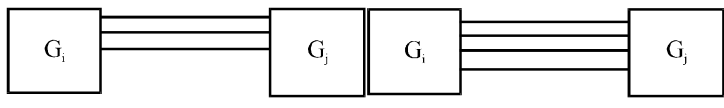

Fig. 2: Example of solving the layout problem
On the basis of sequential algorithms let us develop the algorithm using the matrix of circuits $\mathrm{T}=\left\|\mathrm{t}_{\mathrm{i}}\right\|_{\mathrm{h} \times \mathrm{k} 1}$ where $n$ number of elements. $K_{1}=\max \times k_{i}, i=\overline{1, n}, t_{j j}$ chain number associated with the element $e_{i}$ and its jth terminal. In addition to matrix $\mathrm{T}$, the list of prohibited items is given that can not be in the same subcircuit.

First, the number of elements in each sub-circuit is zero. Then, let us distribute piecemeal the prohibited items. If these elements are not present then we arbitrary pre-distribute elements as to pieces.

Sequentially, we select the lines $e_{i}$ from the matrix $T$ we determine that piece $G_{j}$ in placement into which the element $e_{i}$ gives the smallest increment of the number of connections between the pieces.

We determine the increment of the number of connections $\Delta \mathrm{K}_{\mathrm{j}}^{\mathrm{i}}$ in placing the element $\mathrm{e}_{\mathrm{i}}$ into the piece $\mathrm{G}_{\mathrm{j}}$. First, we consider the condition of appearance of increment as to chain. According to the matrix $\mathrm{T}$ for element $e_{i}$ we construct the auxiliary line $S_{0}^{\mathrm{i}}=1\left\|\mathrm{~S}_{\infty \delta}^{\mathrm{i}}\right\|_{1 \times \mathrm{m}}$ where:

$$
\mathrm{S}_{0 \delta}^{\mathrm{i}}=\left\{\begin{array}{l}
1 \\
0
\end{array}\right.
$$

Then, the first condition increment occurrence as to chain $q$ is the condition that $S_{o q}^{i}=1$, i.e., element $e_{i}$ is associated with the chain $\mathrm{q}$.

The second condition for appearance of increment of the chain $\mathrm{q}$ is the condition that before introduction of element $e_{i}$ in piece $j$ no element of this piece had no connection to the chain q, i.e.:

$$
\mathrm{S}_{\mathrm{jq}}=0, \overline{\mathrm{S}_{\mathrm{jq}}}=1
$$

The third condition is the existence of relations of chain $q$ with elements of other pieces, except for piece $G_{j}$ that is:

$$
\bigcup_{\substack{\mathrm{k}=1 \\ \mathrm{k} \neq \mathrm{j}}}^{1} \mathrm{~s}_{\mathrm{kq}}=1
$$

Then, the presence of increment of connection as to circuit $\mathrm{q}$ will be determined by the Eq. 6 :

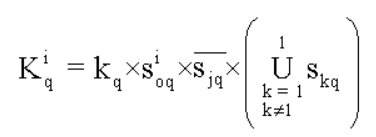

where, $k_{q}$ coefficient of chains equal to the minimum cross-section. For $k_{q}^{i}=1$ there is the increment for $k_{q}^{i}=0$ there is no increments but for all $\mathrm{m}$ circuits: 


$$
\Delta \mathrm{K}_{\mathrm{j}}^{\mathrm{i}}=\sum_{\mathrm{q}=1}^{\mathrm{m}} \mathrm{k}_{\mathrm{q}}\left[\mathrm{s}_{\mathrm{oq}}^{\mathrm{i}} \times \overline{\mathbf{s}_{\mathrm{jq}}} \times\left(\underset{\substack{\mathrm{k}=1 \\ \mathrm{k} \neq \mathrm{j}}}{1} \mathbf{s}_{\mathrm{kq}}\right)\right]
$$

Then, the algorithm is as follows:

1. In matrix $\mathrm{T}$ we select line $\mathrm{e}_{\mathrm{i}}$

2. We build the line $\mathrm{s}_{0}{ }^{\mathrm{i}}$

3. We determine the increments $\Delta \mathrm{k}_{\mathrm{j}}^{\mathrm{i}}, \mathrm{j}=1, \mathrm{p}$ in pieces $1 \div \mathrm{p}$ (or 1 at the first step)

4. We choose $\Delta \mathrm{K}_{\mathrm{j}^{\mathrm{i}}}^{\mathrm{i}}=\min \Delta \mathrm{K}^{\mathrm{i}}$

5. We modify the line ${ }^{j} \mathrm{~S}_{j^{*}}$ of matrix $\mathrm{S}$ by bitwise disjunction with the line $\mathbf{s}_{0}{ }^{\mathrm{i}}$

6. If the number of elements in the piece $G_{j}$ is equal to the given then the piece $G_{j}$ is formed, if it is less then we take the next element and repeat again

Placing the elements and structural assemblies of electrical equipment: The placing problem is to place the elements and structural nodes on the installation space of the vehicle. The problem is formulated as the placement of elements on the set of positions $L=\left\{1_{\mathrm{f}}\right\}, \mathrm{f}=\overline{1, \mathrm{c}}$ of the mounting space. We believe that there are many positions $L=\left\{1_{1}, \ldots, 1_{n}\right\}$ at the object in which will be installed the movable elements. The proposed algorithm is based on sequential algorithms.

Let there be a set of elements $E=\left\{e_{1}, e_{2}, \ldots, e_{n}\right\}$ to locate and plurality of positions $\mathrm{L}=\left\{\mathrm{e}_{1}, \mathrm{e}_{2}, \ldots, \mathrm{e}_{\mathrm{n}}\right\}$ of their installation. At each step of the process $n$ of successive placing is selected one unallocated element and placed into one of the vacant positions.

We determne the rule for choosing the next element and positions for their installation. Let $\mathrm{E}_{\mathrm{k}}$ elements placed the kth step and $\mathrm{L}_{\mathrm{k}}$ the positions taken by these elements; $\overline{E_{k}}$ and $\overline{L_{k}}$ accordingly are unplaced elements and vacant positions. Before placement there may be two situations:

- There are no previously placed elements then the way of selection and installation of the first element should be set

- There is a group of pre-placed elements

As the criterion for optimizing the placement we take the criterion of minimum of the total weight of connections, depending on the length of connections and cross sections of wires. The solution is conducted in two stages:

Choice of element: Choice of element is based on the calculation of the measure of connectivity of still unplaced elements with the placed ones based on adjacency matrix $R=\left\|r_{i j}\right\|_{n \times n}$. The adjacency matrix takes into account the number of links between the elements and parameters of these links (volume of conductive material).

The characteristics of calculation is calculated for each unplaced element of total connectivity with already placed elements:

$$
\mathrm{C}_{\mathrm{i}}=\sum_{\mathrm{e}_{j} \in \mathrm{E}_{\mathrm{k}}} \mathrm{r}_{\mathrm{ij}}, \mathrm{e}_{\mathrm{i}} \in \overline{\mathrm{E}_{\mathrm{k}}}
$$

To select the element it is also necessary to calculate the amount of conductor material of connections, depending on the number of links, wire cross section and length of the wire:

$$
\mathrm{r}_{\mathrm{ij}}=\sum_{\mathrm{s} \in \mathrm{J}_{\mathrm{ij}}} \mathrm{k}_{\mathrm{q}} \times \omega_{\mathrm{s}}
$$

Where:

$\mathrm{J}_{\mathrm{ij}}=$ The set of circuits connecting elements $\mathrm{e}_{\mathrm{i}}$ and $\mathrm{e}_{\mathrm{j}}$

$\omega_{s}=$ Weight coefficient

$\mathrm{k}_{\mathrm{q}}=$ Coefficient equal to the minimum cross section

Select the element with the largest number of connections with already placed elements, i.e., $\mathrm{e}_{\mathrm{i} *} \Rightarrow \mathrm{C}_{\mathrm{i}^{*}}=\max \mathrm{C}_{\mathrm{i}}$.

Positioning: The selected element is located in one of the vacant positions of the array $\mathrm{L}_{\mathrm{n}}$. This position is selected according to the criterion of minimum of total length of the connections. We use orthogonal metric of three-dimensional mounting space of the vehicle:

$$
F_{S}=\sum_{V_{q} \in V\left(i, E_{k}\right)}\left[\left(x_{s \text { max }}^{q}-x_{s \min }^{q}\right)+\left(y_{s \max }^{q}-y_{s \min }^{q}\right)+\left(z_{s \max }^{q}-z_{s \min }^{q}\right)\right]
$$

The position is selected as to $\mathrm{F}_{\mathrm{S}}=\min$. The rule of selection of the first element (if there are no placed elements or): such element serves as the center, around which the other elements are grouped. Typically the element is searched with the greatest number of connections with the other element $r_{i}=\sum_{e_{i} \in E} r_{i j}, r_{i^{*}}=\max r_{i}$
and it is placed in the central position.

Formation of bunched wires configuration: As a result of solving the problems of layout and placement of the structural units and electrical circuits wiring we have the non-overlapping sets of wires, each of which implements the single chain. Wires are usually combined into bunches, together forming the wiring diagram. The conditional picture of bunched wire which is the two-dimensional scan of three-dimensional model of the bunched wire is shown in Fig. 3 . 


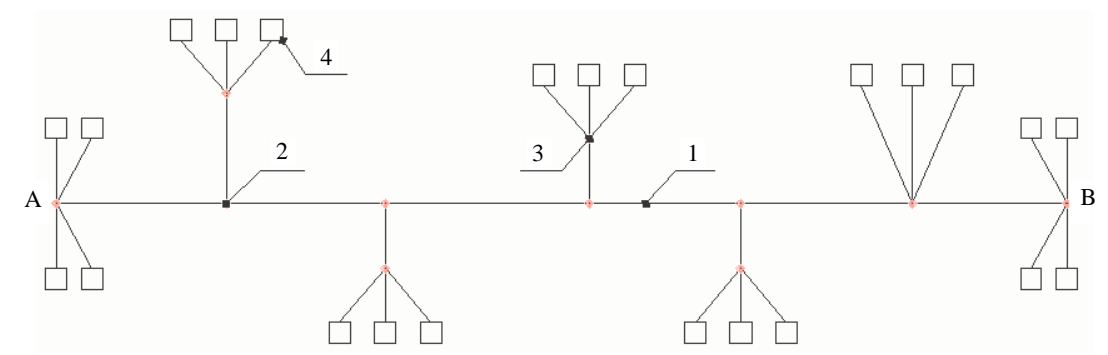

Fig. 3: Conditional picture of bunched wire; 1: A-B trunk of bunched wire; 2: branches; 3: branching points of the group of wires and 4: connectors

For a certain number and location of connectors, set of entering the bunched wire conductors the task of synthesis of electric bunched wire is to determine its configuration, being the finding the optimal number, composition and location of branches and implementation of restrictions on diameters of individual sections of the trunk and branches of the bunched wire. At the same time, we assume that branches go away from the trunk at right angles. This assumption is justified, since the final angle of inclination of branches is determined after the stages of prototyping and implementation of pre-production model of electrical mounting on the car.

In addition, if there are no restrictions on the lining route of branching then it is selected as a rule as to the shortest path, i.e., perpendicular to the trunk of bunched wire. With the foregoing assumption and selected orthogonal metric in designing the mounting space of the vehicle the points 3 of branching of wire group coincide with the points of branching 2 (as shown for some branches in Fig. 2). In addition, taking into account that the plurality of connecting devices of individual branches do not intersect, further we will consider without loss of generality, only a portion of the bunched wire.

In solving the optimization problem of forming the bunched wires the value of target function $F_{2}$ is the sum of indicators of quality of individual branches:

$$
\begin{aligned}
& \mathrm{F}_{\mathrm{bw}}=\sum_{\mathrm{p}=1}^{\mathrm{m}} \mathrm{F}_{\mathrm{p}} \rightarrow \min \\
& \mathrm{F}_{\mathrm{p}}=\sum_{\mathrm{k} \in \mathbb{N p}}\left(\mathrm{x}_{\mathrm{k}}+\mathrm{y}_{\mathrm{k}}\right) \times \mathrm{s}_{\mathrm{k}}
\end{aligned}
$$

Where:

$$
\begin{aligned}
& \mathrm{F}_{\mathrm{p}}=\text { Quality factor which determines the total volume } \\
& \text { of conductive material of pth branch } \\
& \mathrm{N}_{\mathrm{p}} \quad=\text { Plurality of conductors of pth branch } \\
& \mathrm{x}_{\mathrm{k}}, \mathrm{y}_{\mathrm{k}}=\text { Coordinates of the connecting device of } \mathrm{kth} \\
& \text { wire }
\end{aligned}
$$

$\mathrm{s}_{\mathrm{k}}=$ Cross section of the kth wire which is determined $\mathrm{s}_{\mathrm{k}}=\max \left(\mathrm{s}_{\mathrm{k}}, \mathrm{s}_{\mathrm{knex},}, \mathrm{s}_{\mathrm{ka} 3}\right)$ as here $\mathrm{s}_{\mathrm{kH}}, \mathrm{s}_{\mathrm{kneex}}, \mathrm{s}_{\mathrm{ka} 3}$ are minimum cross sections of the kth wire, selected, respectively from the conditions of allowable values for heating, mechanical strength and protection ability of protection devices

To simplify, we assume that $\mathrm{s}_{\mathrm{k}}=1$, does not affect the technique of solving the problem. Restrictions at the optimum synthesis of bunched wires are:

$$
\mathrm{D}_{\text {omb.p }} \leq \mathrm{D}_{\text {omb.jonp, }} \mathrm{p}=\overline{1, \mathrm{~m}}
$$

$D_{\text {cmb.p. }}, D_{\text {omb.romp }}$ diameter of conductors group of pth branch and its allowable value:

$$
\mathrm{D}_{\text {cmb.p }} \leq \mathrm{D}_{\text {cmb.əonp, }} \mathrm{p}=\overline{1, \mathrm{~m}}
$$

$D_{\text {cmb.p. }}, D_{\text {cmb.on. }}$ diameter of the trunk of bunched wire between " $\mathrm{p}$ "-th and “p-1»--th branches and its allowable value. Values $D_{\text {om } \beta \text { p }}, D_{\text {cm } \beta p}$ are determined as to nomograms depending on the number of conductors and their diameters.

The total diameter of the wire is determined as follows. For wires with identical outer diameters the total diameter is determined by the Eq. 15 :

$$
\mathrm{D}=\mathrm{c} \times \mathrm{d}
$$

Where:
$\mathrm{c}=$ The coefficient depending on the number of wires in the bunched wire

$\mathrm{d}=$ Outer diameter of the wire

For wires with different outer diameters, the total diameter is determined by the Eq. 16 :

$$
\mathrm{D}=1.1 \times \mathbf{c} \times \mathrm{d}_{\ni}
$$

where, $d_{3}$ is equivalent outer diameter of the wires: 


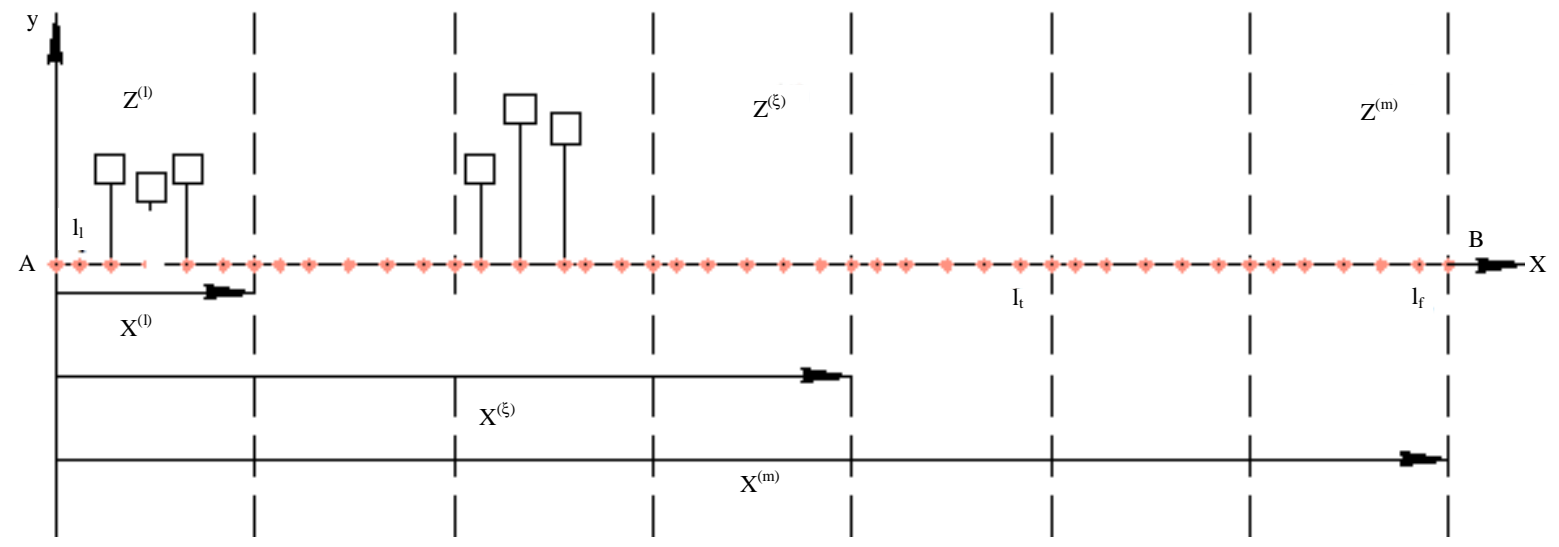

Fig. 4: Model of mounting plane of bunched wire

$$
\mathrm{d}_{3}=\sqrt{\frac{\mathrm{d}_{1}^{2}+\mathrm{d}_{1}^{2}+\ldots+\mathrm{d}_{\mathrm{n}}^{2}}{\mathrm{n}}}
$$

Where:

$$
\begin{array}{ll}
\mathrm{n} & =\text { The number of wires } \\
\mathrm{d}_{1}, \mathrm{~d}_{2}, \ldots, \mathrm{d}_{\mathrm{n}}=\text { Diameters of wires }
\end{array}
$$

To solve the Eq. 12-15, we will use the length property of the design object as to coordinate $\mathrm{x}$. In the decision procedure, it is proposed approximate method in which the initial problem is viewed as a multi-step decision-making process. This requirement is satisfied our task after ordering as to the coordinates along the axis $\mathrm{x}$ of the array of positions set to place the branch points of the bunched wire and array of connecting devices. For organization of computational process we divide the mounting plane of the bunched wire by cutting planes perpendicular to the axis $\mathrm{x}$ and determine the array of zones (Fig. 4):

$$
\mathrm{Z}=\left\{\mathrm{Z}^{(\mathrm{\xi})}: \mathrm{x}^{(\mathrm{\xi})}=\frac{\xi \times \mathrm{x}_{\mathrm{m}}}{\mathrm{m}} ; \mathrm{x}_{0}^{(\mathrm{\xi})}=0 ; \xi=\overline{1, \mathrm{~m}}\right\}
$$

Where:

$\mathrm{x}_{0}^{(\xi)}, \mathrm{x}^{(\xi)}=$ Starting and ending coordinates of the cutting planes of the $\xi$ th zone

$\mathrm{x}_{\mathrm{m}}=$ Limiting coordinate of the mounting plane

Suppose that a set of connecting devices $N_{q}=\left\{q_{1}, \ldots\right.$, $\left.\mathrm{q}_{\mathrm{s}}, \ldots, \mathrm{q}_{\mathrm{n}}\right\}$ ordered as to coordinate $\mathrm{x}$ connected by conductors with the branch points and a set of positions $\mathrm{L}=\left\{1_{1}, \ldots, 1_{\mathrm{t}}, \ldots, \mathrm{l}_{\mathrm{f}}\right\}$ to locate the branch points from the set $\mathrm{P}=\left\{\mathrm{p}_{1}, \ldots, \mathrm{p}_{\mathrm{j}}, \ldots, \mathrm{p}_{\mathrm{n}}\right\}$. The set of positions $\mathrm{L}=\left\{1_{1}, \ldots, \mathrm{l}_{\mathrm{t}}, \ldots\right.$, $\left.1_{\mathrm{f}}\right\}$ is formed by the trunk points, evenly or unevenly spaced (by decision of the designer) between the cutting planes. The step between positions is selected depending on the desired accuracy and computational capabilities of the computer used.
At the first step of the algorithm, we determine the best position to place the first branch point sequentially in zones $\mathrm{Z}^{(\xi)}, \xi=\overline{1, \mathrm{~m}}$ and from it we conditionally “attach» the wires from connectors located in these areas. Thus, we find the value of target function:

$$
\mathrm{F}_{1}=\min _{1_{\mathrm{t}} \in \mathrm{L}} \sum_{\mathrm{k} \in \mathbb{N}_{1}^{\xi}}\left(\mathrm{x}_{\mathrm{k}}+\mathrm{y}_{\mathrm{k}}\right) \times \mathbf{s}_{\mathrm{k}}
$$

where, $\mathrm{N}_{1}^{\xi}$ subset of connectors located in the zone $Z^{(\xi)}$. At jth step recursion expression is used:

$$
F_{j}(\xi)=\min _{\psi}\left[F_{j}(\xi, \psi)+F_{j-1}(\psi)\right], \xi=\overline{j, m} ; \psi=\overline{j-1, \xi-1}
$$

where, $F_{j-1}(\xi)$ the optimal value of the target function computed at the previous step, when placing $j-1$ branches in the zone $Z(\psi)$.

The value $F_{j}(\xi, \psi)$ of target function when placing the jth branch in the zone $Z_{\xi}$ with coordinates $x^{(4)} \leq x(\xi)<x^{(\xi)}$ and attaching the jth branch to connectors with these coordinates.

Each jth step of the computational process corresponds to the formation of $\mathrm{j}$ branches and determination of their composition as to connecting devices using all $\mathrm{m}$ zones. Following $\mathrm{n}$ steps we will have $\mathrm{n}$ options of synthesis of bunched wire configuration, among them we choose the option with the least value of the target function. Then the reverse is performed, resulting in determination of location of each branch and composition of its constituent connectors.

\section{CONCLUSION}

The developed technique of computer-aided design of power supply system of the vehicle including the 
layout and placement of structural units of electrical equipment, wiring of electrical circuits, forming and tracing the bunched wires, optimizing the cross-sections of wires, choice of protective devices, electrical energy balance calculation, analysis of operation modes allows one to determine the optimal number of electric structures, optimum wiring of electrical circuits to determine the correctness of selection of wire cross sections, protection devices, power of generator and battery according to different modes of operation of power supply system of the vehicle. Commercial operation of this technique allows us to reduce design time by $8-12 \%$, decrease the mass of wiring by $3-5 \%$, reduce errors in design of power supply system of the vehicle.

\section{ACKNOWLEDGEMENTS}

The research is done by “Kazan National Research Technical University named after A.N. Tupolev-KAI» with financial support of the Ministry of Education and Science of the Russian Federation, the unique identifier for applied scientific research (project) RFMEFI57414X0050.

\section{REFERENCES}

Fedorov, E.Y., V.S. Tereshchuk and A.V. Ferenetz, 2015. Wiring of complicated electrical circuits of interblock assembling in automated design of aircraft. Russ. Aeronautics, 58: 96-99.

Hart, P.E., N.J. Nilsson and B. Raphael, 1968. A formal basis for the heuristic determination of minimum cost paths. IEEE Trans. Syst. Cybernet., 4: 100-107.

Lee, C.Y., 1961. An algorithm for path connections and its applications. Electron. Comput., IRE Trans., 3: $346-365$.

Norenkov, I.P., 2002. Information support of high-tech products. CALS Technol. 